\title{
The impact of logarithmic dose banding of anticancer drugs on pharmacy compounding efficiency at Ghent University Hospital
}

\author{
Barbara Claus, ${ }^{1,2}$ Kaat De Pourcq, ${ }^{3}$ Nele Clottens, ${ }^{1}$ Vibeke Kruse, ${ }^{4}$ Paul Gemmel, ${ }^{3}$ \\ Johan Vandenbroucke ${ }^{1}$
}

1 Department of Pharmacy, Ghent University Hospital, Ghent, Belgium

${ }^{2}$ Faculty of Pharmaceutical Sciences, Ghent University, Ghent, Belgium

${ }^{3}$ Faculty of Economics and Business Administration, Ghent University, Ghent, Belgium ${ }^{4}$ Department of Medical Oncology, Ghent University Hospital, Ghent, Belgium

\section{Correspondence to} Dr Barbara Claus, Ghent University Hospital, Pharmacy department, K12,-1 De Pintelaan 185, Ghent 9000, Belgium; Barbara.claus@ ugent.be

Received 25 August 2016 Revised 10 January 2017 Accepted 23 January 2017 Published Online First

4 March 2017

EAHP Statement 3 Production and Compounding

A Check for updates

To cite: Claus B, Pourcq KD,

Clottens N, et al.

Eur J Hosp Pharm

2018:25:334-336

\begin{abstract}
Background Dose banding (DB) (dose rounding with predetermined variation with prescription) enables inadvance preparation of high-turnover anticancer drugs with potential benefit for pharmacy compounding work flow. Objectives To analyse the impact of potential situations on the efficiency of DB in the pharmacy (safe and maximum storage), calculate preparation lead times and the potential full-time equivalent (FTE) benefit.

Methods Candidate intravenous anticancer drugs were selected for logarithmic DB according to prescribing frequency, infusion volume and stability (usage data 2015 of the tertiary Ghent University Hospital, Belgium). With a selected DB set already stored, a 2-week time study (April/ November 2015) provided lead times (between prescription and transfer) for just-in-time and DB preparations. A 'maximal' storage (using all drugs with a relative incidence of $\geq 2 \%$ recurrent monthly prescription) and a 'safe' storage scenario (lowest monthly prescribing pattern) were used to calculate the potential future FTE change.

Results Mean lead times for DB storage and just-in-time preparation were $17.1 \mathrm{~min}(95 \% \mathrm{Cl} 13.5$ to 21.0$)$ and 26.5 $\min$ (23.3 to 29.8). For 21164 yearly preparations with already 5292 in DB (25\%), 11157 and 6862 could be batch-produced in advance in a maximum storage and safe storage scenario, respectively. The existing FTE in 2015 of 5.41 could then be reduced to 4.91 and 5.27.

Conclusion Further development of DB could contribute to pharmacy compounding efficiency.
\end{abstract}

\section{INTRODUCTION}

The concept of dose standardisation, further mentioned as dose banding (DB), has been introduced to rationalise the prescribed dose of intravenous (IV) anticancer drugs for the following reasons: (1) to make some preparations in advance in centralised IV additive services) or compounding centres and (2) to accelerate in-hospital turnaround time for commonly used preparations, contributing to enhanced daily patient capacity in oncology clinics.

The key principle of DB was introduced by Plumridge and Sewell ${ }^{2}$ as the preparation of standardised doses with a predetermined maximum variation between prescribed and prepared dose. A widely accepted method to round prescriptions up or down to standardised doses is logarithmic dose banding (LDB), described by Geoff Hall. LDB provides bands with dose reductions consistent between all bands and uses a maximum deviation of $5.8 \%$ from the original prescription. By agreement the mid-dose of a band is prepared. LDB has been adapted by many cancer groups, such as the Peninsula Cancer Network ${ }^{3}$ and the Scottish Oncology Pharmacy Group, ${ }^{4}$ and is being gradually implemented in many oncology centres.

Consequently, a range of infusions, manufactured by pharmacy staff, can be stored for direct delivery on demand. These infusions must remain stable and have sufficient turnover. New guidelines for stability testing of anticancer treatments have been published ${ }^{6}$ and data were updated with new biotechnological medicinal oncology products, leading to comfortable shelf lives of several days up to months.

The economic impact of DB at pharmacy level, however, has not been described. In Belgium, no standard requirement exists to implement DB but for efficiency, many hospitals adopt a phased implementation of the standard LDB list of Geoff Hall for individually chosen high-turnover molecules. In 2015, the 1000-bed Ghent University Hospital, situated on one campus in Belgium, gradually implemented DB storage of five high-turnover molecules: 5-fluorouracil, oxaliplatin, gemcitabine, paclitaxel and rituximab. This article describes the situation in 2015 and the potential benefit of LDB for the pharmacy work flow by expansion with more candidate molecules for LDB. It aims to analyse the impact of potential scenarios on the efficiency of the process in the pharmacy (a safe storage and a maximum storage scenario), calculate preparation lead times and the potential full-time equivalent (FTE) benefit. We provide some practice recommendations.

\section{METHODS}

The following usage data for all prescribed biological and cytotoxic anticancer drugs (non-paediatric/not clinical trial related) in 2015 were retrieved from the electronic oncology prescribing system (Chemopro 2.0, in-house software) of the Ghent University Hospital, Belgium: international non-proprietary name, dose, final concentration after dilution in a specified solvent and day of preparation. In 2015, LDB of 5-fluorouracil, oxaliplatin, gemcitabine, paclitaxel and rituximab provided a secured storage of 25 different dose strengths. Simulation of more candidate molecules was carried out to explore the potential future benefit.

First, a 2-week study (two separate weeks in April and November 2015) was performed by the Centre of Service Intelligence of Ghent University (Faculty of Economics and Business Administration) to collect lead times of prescriptions and calculate differences between stored DB and just-in-time preparations. 
Lead time was defined as the time between receipt of a prescription and readiness for transfer, reported as mean ( $\mathrm{min}$ ) with 95\% CI. It should be noted that several preparations can pass through the system at the same time. Transfer started once the preparation was individually labelled and placed at the supply chain department picking desk (with a 15 min pick-up frequency) or sent through a pneumatic tubing system throughout the hospital.

Selection of candidate molecules eligible for DB was based on a physicochemical stability of at least 7 days (combined data from the literature and manufacturer), the frequency of prescribing and the infusion volume (ie, final concentration). A total number of potential DB molecules was calculated for 2015, estimating future storage. Data are expressed as the number of different DB strengths and the total number of stored DB preparations versus just-in-time preparations. Data are analysed both for 2015 and future storage.

Two future scenarios are presented:

1. A 'maximum' storage scenario: all preparations in 2015 were rearranged per band and only stable DB preparations (midband-doses) with a relative incidence of at least $2 \%$ recurrent monthly prescription were retained. This maximum scenario includes the currently stored LDB molecules chosen based on the same criteria.

2. A 'safe' storage scenario using the conditions mentioned in point 1 but further corrected for the lowest prescribing amount within the documented shelf-life and calculated per month. For a product with a stability of, for example, 1 month, only the smallest number prescribed in the previous 12 months was retained.

By combining the time study with future storage possibilities, a difference in pharmacy working hours (FTE) could be estimated between the actual situation and the future forecast. The mean preparation time of a DB batch was incorporated.

\section{RESULTS}

In the 2-week study a total of 888 individual prescriptions were analysed of which $94(10.6 \%)$ were stored as DB. The mean lead times for DB storage and just-in-time preparations respectively were $17.3 \mathrm{~min}$ (95\% CI 13.5 to 21.0 ) and $26.5 \mathrm{~min}$ (23.3 to 29.8).

Of the 39 anticancer drugs (10 monoclonal antibodies (mAbs)/ cytokines and 29 cytotoxic agents/diverse antitumoral drugs) 15 IV anticancer drugs had stability data of at least 7 days and monthly prescriptions: bevacizumab, carboplatin, cisplatin, docetaxel, epirubicin, 5-fluorouracil, gemcitabine, irinotecan, methotrexate, oxaliplatin, paclitaxel, pemetrexed, rituximab, IV trastuzumab and vincristine.

The overall number of anticancer prescriptions in 2015 was 21164 (approximately 58/day) of which 5292 were already stored as DB (25.0\%). According to the simulation 6862 ('safe' scenario) up to 11157 preparations ('maximum' scenario) could be batch produced in advance. This entails an expected storage of $32.4 \%$ (19/day) to a maximum of $52.7 \%$ (31/day) of all daily prescriptions. In total 85 different strengths can be stored with a stability varying between 7 days (vincristine) and 6 months (trastuzumab). An overview is given in table $1 .^{7-12}$

The mean time of preparation within a DB batch was $0.99 \mathrm{~min}$ and $2.56 \mathrm{~min}$ for, respectively, one infusion bag and one infusion pump.

Compared with 2015 with 8651.5 (7468.8-9849.8) pharmacy working hours per year or $5.41 \mathrm{FTE}$, the two scenarios had the following impact: the safe storage scenario required an estimated

Table 1 Storage possibilities in 2015 and the future applying logarithmic dose banding of intravenous anticancer drug preparations with usage data of 2015 in a tertiary care hospital

\begin{tabular}{|c|c|c|c|c|c|c|}
\hline INN & $\begin{array}{l}\text { Situation in } 2015 \\
\text { No. of individual } \\
\text { prescriptions in } \\
2015 \\
\text { per product } \\
\text { (n=21 164) }\end{array}$ & $\begin{array}{l}\text { Real-life storage } \\
\text { No. of } \\
\text { DB strengths in } \\
2015 \\
(n=25)\end{array}$ & $\begin{array}{l}\text { Future storage } \\
\text { No. of potential } \\
\text { DB strengths per } \\
\text { year } \\
(n=85)\end{array}$ & $\begin{array}{l}\text { Maximal future } \\
\text { storage } \\
\text { No. of } \\
\text { DB preparations } \\
(\mathrm{n}=21 \text { 164) }\end{array}$ & $\begin{array}{l}\text { Safe future } \\
\text { storage } \\
\text { No. of } \\
\text { DB preparations } \\
(n=21164)\end{array}$ & $\begin{array}{l}\text { Shelf life } \\
\text { under specific } \\
\text { conditions } \\
\text { in days }^{\text {ref }}\end{array}$ \\
\hline Bevacizumab & 665 & 0 & 9 & 554 & 333 & $90^{7}$ \\
\hline Carboplatin & 1045 & 0 & 12 & 752 & 368 & $56^{78}$ \\
\hline Epirubicin & 406 & 0 & 4 & 373 & 217 & $84^{*}$ \\
\hline $\begin{array}{l}\text { 5-Fluorouracil } \\
\text { infusion bag }\end{array}$ & 1957 & 5 & 5 & 949 & 600 & $28^{*}$ \\
\hline pump & 1038 & 3 & 6 & 1466 & 978 & \\
\hline Gemcitabine & 830 & 5 & 9 & 775 & 465 & $84^{*}$ \\
\hline Pemetrexed & 162 & 0 & 3 & 133 & 48 & $31^{10}$ \\
\hline Rituximab & 600 & 3 & 5 & 552 & 348 & $90^{11}$ \\
\hline Trastuzumab & 1089 & 0 & 4 & 1019 & 920 & $180^{12}$ \\
\hline Vincristine & 934 & 0 & 1 & 427 & 313 & $7^{7}$ \\
\hline $\begin{array}{l}\text { Summation DB, } \mathrm{n} \text { (\% of all individual } \\
\text { prescriptions) }\end{array}$ & $5292(25.0)$ & NA & NA & $11157(52.7)$ & $6862(32.4)$ & \\
\hline $\begin{array}{l}\text { Summation preparations NOT DB, n (\% } \\
\text { of all individual prescriptions) }\end{array}$ & $15872(75.0)$ & NA & NA & $10007(47.3)$ & 14302 (67.6) & \\
\hline
\end{tabular}

*Data obtained from manufacturer.

DB , dose band; INN, International non-proprietary name; NA, not applicable. 
8434.0 (7236.7-9643.8) hours per year or 5.27 FTE and the maximum storage scenario 7859.1 (6618.9-9097.6) working hours per year or 4.91 FTE.

\section{DISCUSSION}

This study indicates a potential change of LDB on the pharmacy organisation, with an estimated average yearly reduction of 0.5 FTE when the maximal potential storage scenario is compared with the situation in 2015. However, we believe that it is best to start with the conservative 'safe' scenario, given the storage of high-cost drugs such as mAbs. The benefit-risk balance needs to be borne in mind: ready-to-use storage versus risk of expiry of the stored bands. It is worth mentioning that literature simulations showed a cost avoidance when using dose rounding of high-cost biological agents or through making DB batch productions (fewer fractions lost). ${ }^{13} 14$ Cost avoidance through DB production evidently assumes that expiry on storage is not occurring.

An important criticism is that the dose of intravenous anticancer drugs used depends on body surface area (BSA). The correlation between the area under the curve (AUC) of the plasma concentration over time (which is linked to toxicity and efficacy) and BSA is rather poor. This ascertainment could undermine the acceptance of DB built on BSA dosing. Regardless of the fact that some authors have shown no significant difference in precision (AUC versus target AUC in pharmacokinetic models) between using DB ranges and classic BSA dosing, the inaccuracy of BSA remains an important concern. ${ }^{15}$ This cannot be solved with $\mathrm{DB}$.

The real advantage of efficient preparation in oncology is the subcutaneous formulation of mAbs such as rituximab and trastuzumab. These formulations are not included in the above calculations, but today, two out of three preparations of trastuzumab in our centre are administered subcutaneously.

It is further important to state that the used stability data are strictly linked to the used brand name, infusion volume, final concentration and manufacturer's data which can all differ between centres.

We make the following future recommendations to other European centres:

- Some shelf lives exceed 3 months, therefore, it is strongly advised from a microbiological aspect that sterility testing should be in place. Our hospital simultaneously uses a validated BacT/Alert 3D microbial detection system (Biomérieux, Durham, USA) to test microbiological stability.

- Before making a new batch of a selected DB strength, an immediate re-evaluation of usage is needed (eg, previous 3 months), correcting for drug use fluctuation over time. This could be carried out with an automated query and protects against expiry.

- We recommend that physicians are clearly informed about which drugs are considered for DB. In our electronic system molecules following LDB are directly converted to mid-dose on prescribing. Physicians are alerted and can over-rule the dose standardisation proposal. This method also enables automatic exclusion of clinical trials and paediatric prescriptions.

- With an integrated embedding of the LDB dose in the electronic system there is no confusion due to a difference between the prescribed dose and labelling on the delivered infusion bag.

- The storage room should be large enough, with 24-hour monitoring in a refrigerator and at room temperature. To store the 'maximum' scenario, we expect that two extra refrigerators will be needed. In a cost-benefit analysis, the cost of storage should also be considered.

We do not expect an FTE reduction at the preparation level to follow from the present DB implementation. Inclusion of more molecules into the $\mathrm{DB}$ will enable us to save on preparation time, which will then be used for better quality assurance. Our hospital pharmacy investigates in-process controlled systems (eg, with Intravenous SOFT Assist (Aesynt Incorporated, Italy), Isimix (Isitec Srl, Italy), Medimix (Impromediform, $\mathrm{GmbH}$ ), Cato (BD Medical), etc), ensuring complete monitoring of each preparation step. This quality project substantially expands preparation time. We believe that a better spread of the pharmacy work through DB enables a more homogeneous occupation of the CIVA personnel, resulting in better work with the same or fewer personnel. Reduction of the just-in-time preparations in our centre is needed to cover the $5-10 \%$ increase in oncological treatments each year.

As a final goal, DB contributes to reduced waiting time in day clinics in addition to other efforts, such as laboratory parameter checks on the day before consultation. If a drug forms part of a chemotherapy doublet or triplet, we believe that the benefit from $\mathrm{DB}$ will be the greatest if all drugs of the set are prepared ahead.

Acknowledgements We acknowledge Danielle Delaey, master student in pharmaceutical sciences of Ghent University Hospital, for the preliminary analyses on dose banding in her master dissertation in 2016.

\section{Competing interests None declared.}

Provenance and peer review Not commissioned; externally peer reviewed.

(C) European Association of Hospital Pharmacists (unless otherwise stated in the text of the article) 2018. All rights reserved. No commercial use is permitted unless otherwise expressly granted.

\section{REFERENCES}

1 Hoppe-Tichy T. Current challenges in European oncology pharmacy practice. J Oncol Pharm Pract 2010;16:9-18.

2 Plumridge RJ, Sewell GJ. Dose-banding of cytotoxic drugs: a new concept in cancer chemotherapy. Am J Health Syst Pharm 2001;58:1760-4.

3 Hardy L, Cox M, Peninsula Cancer Network ChemoCare Consortium 247 Trusts. Logarithmic dose banding proposal and implementation for 248 chemotherapy. A Document Published by the National Dose Banding 249 Working Party of the Peninsula Cancer Network, Devon and Cornwall, of 250 National Health Service NHS,UK. 2012.

4 Scottish Oncology Pharmacy Group (SOPPG). Guidelines for dose banding of systemic Anti-Cancer therapy development and governance framework. 2015.

5 Pouliquen AL, Escalup L, Jourdan N, et al. Dose standardisation of anticancer drugs. Int J Clin Pharm 2011;33:221-8.

6 Bardin C, Astier A, Vulto A, et al. French Society of Oncology Pharmacy. Guidelines for the practical stability studies of anticancer drugs: a European consensus conference. Ann Pharm Fr 2011:69:221-31.

7 Vigneron J, Astier A, Trittler R, et al. French Society of Hospital Pharmacists; European Society of Oncology Pharmacists. SFPO and ESOP recommendations for the practical stability of anticancer drugs: an update. Ann Pharm Fr 2013;71:376-89.

8 https://medicines.org.uk [ accessed 10th July 2016]

9 Eiden C, Philibert L, Bekhtari K, et al. Physicochemical stability of oxaliplatin in 5\% dextrose injection stored in polyvinyl chloride, polyethylene, and polypropylene infusion bags. Am J Health Syst Pharm 2009;66:1929-33.

10 Zhang Y, Trissel LA. Physical and chemical stability of pemetrexed in infusion solutions. Ann Pharmacother 2006:40:1082-5.

11 Paul M, Vieillard V, Jaccoulet $E$, et al. Long-term stability of diluted solutions of the monoclonal antibody rituximab. Int J Pharm 2012;436:282-90.

12 Paul M, Vieillard V, Da Silva Lemos R, et al. Long-term physico-chemical stability of diluted trastuzumab. Int J Pharm 2013;448:101-4.

13 Vandyke TH, Athmann PW, Ballmer CM, et al. Cost avoidance from dose rounding biologic and cytotoxic antineoplastics. J Oncol Pharm Pract 2016 (Epub ahead of print).

14 Vandenbroucke J, Robays H. Economic impact of the preparation scenario for cytotoxic drugs: an observational study. Eur J Hosp Pharm Sci Pract 2008;14:37-42.

15 Chatelut E, White-Koning ML, Mathijssen RH, et al. Dose banding as an alternative to body surface area-based dosing of chemotherapeutic agents. $\mathrm{Br} J$ Cancer 2012;107:110-6. 